\title{
TABLE DES MATIÉRES
}

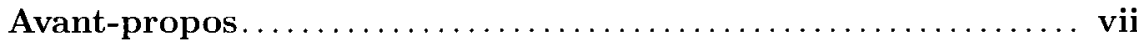

Analyse du contenu du livre......................... viii

Organisation du livre......................... xi

Préambule sur l'ellipticité $\ldots \ldots \ldots \ldots \ldots \ldots \ldots \ldots \ldots \ldots \ldots \ldots \ldots$

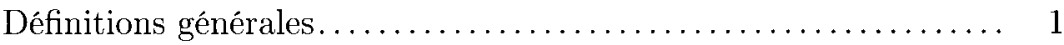

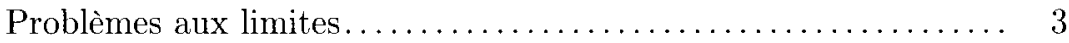

Équations non traitées dans le cadre de ce cours............. 5

1. Rappels de topologie et d'analyse fonctionnelle........ 7

1.1. Espaces vectoriels topologiques..................... 7

1.2. Formes linéaires, dual topologique, topologie faible........ 14

1.3. Espace des fonctions continues sur un ouvert de $\mathbb{R}^{N} \ldots \ldots 26$

1.4. Distributions sur un ouvert de $\mathbb{R}^{N} \ldots \ldots \ldots \ldots \ldots \ldots \ldots \ldots .29$

1.5. Espaces $L^{p}$, lorsque $p \in[1,+\infty] \ldots \ldots \ldots \ldots \ldots \ldots \ldots \ldots \ldots . \ldots \ldots$

1.6. Exercices sur le chapitre $1 \ldots \ldots \ldots \ldots \ldots \ldots \ldots \ldots \ldots \ldots . \ldots \ldots$

2. Les espaces de Sobolev. Théorèmes d'injection..........61

2.1. Définitions et premières propriétés................... 61

2.2. Injections de Sobolev pour $W^{m, p}\left(\mathbb{R}^{N}\right) \ldots \ldots \ldots \ldots \ldots \ldots \ldots 72$

2.3. Généralisation à d'autres ouverts................. 87

2.4. Injections compactes lorsque l'ouvert est borné.......... 98

2.5. Trace sur la frontière d'un ouvert $\mathcal{C}^{1} \ldots \ldots \ldots \ldots \ldots \ldots \ldots \ldots$



3. Traces des fonctions des espaces de Sobolev ...........117

3.1. Espaces $W^{1-1 / p, p}\left(\mathbb{R}^{N-1}\right)$, pour $p>1 \ldots \ldots \ldots \ldots \ldots \ldots \ldots \ldots \ldots$

3.2. Cas du bord d'un ouvert autre que $\left.\mathbb{R}^{N-1} \times\right] 0, \infty[\ldots \ldots \ldots 133$

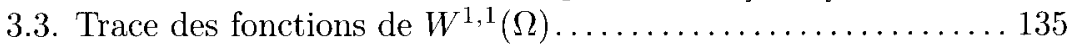

3.4. Densité de $\mathcal{C}^{1}(\partial \Omega)$ dans $W^{1-1 / p, p}(\partial \Omega) \ldots \ldots \ldots \ldots \ldots \ldots \ldots 137$

3.5. Traces d'ordre supérieur........................... 148

3.6. Théorèmes d'injections continues. Injections compactes..... 166

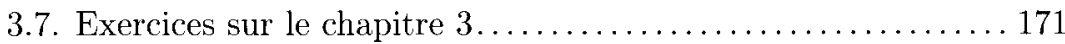


4. Espaces de Sobolev fractionnaires.................. 181

4.1. Distributions tempérées et transformation de Fourier...... 181

4.2. Les espaces de Sobolev $H^{s}\left(\mathbb{R}^{N}\right) \ldots \ldots \ldots \ldots \ldots \ldots \ldots \ldots \ldots$

4.3. Les espaces $W^{s, p}(\Omega)$ pour $0<s<1 \ldots \ldots \ldots \ldots \ldots \ldots \ldots \ldots 1$

4.4. Théorèmes d'injection pour les $W^{s, p}(\Omega) \ldots \ldots \ldots \ldots \ldots \ldots 212$

4.5. Injections compactes pour les $W^{s, p}(\Omega), \Omega$ borné ......... 218

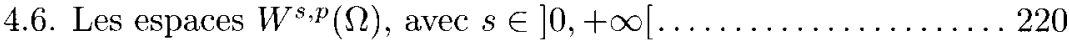

4.7. Appendice : théorème de convexité de Riesz............. 222



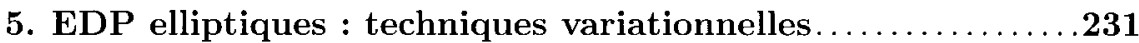

5.1. Présentation de quelques résultats utiles............. 231

5.2 . Rappels d'analyse convexe..................... 232

5.3. Résolution d'EDP linéaires elliptiques de type Dirichlet.... . 238

5.4. Régularité des solutions précédentes................. 245

5.5. Problèmes de Neumann. . . . . . . . . . . . . . . . . . . . 253

5.6. Problèmes de Dirichlet et de Neumann non homogènes. . . . . 260

5.7. Problème de l'élasticité......................... 262

5.8. L'équation du $p$-laplacien.......................... 264

5.9. Principes du maximum pour des EDP elliptiques......... 268

5.10. Problèmes coercifs sur des espaces non réflexifs.......... 283

5.11. Surfaces minimales................................. 285

5.12 . Exercices sur le chapitre $5 \ldots \ldots \ldots \ldots \ldots \ldots \ldots \ldots \ldots \ldots \ldots$

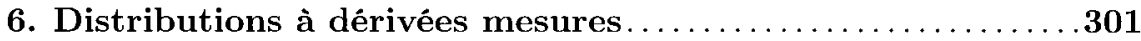

6.1. Rappels sur les mesures, convergences................ 302

6.2. Extension d'une mesure positive ................... 308

6.3. Espace de fonctions à variation bornée................ 316

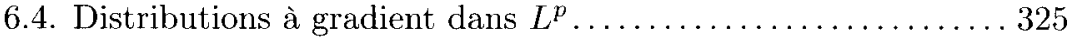

6.5. Distributions à gradient dans $M^{1}(\Omega) \ldots \ldots \ldots \ldots \ldots \ldots \ldots \ldots \ldots \ldots \ldots \ldots$

6.6. Fonctions à déformations dans $L^{p}$, avec $1<p<\infty \ldots \ldots \ldots 328$

6.7. Espaces de fonctions à déformation dans $L^{1} \ldots \ldots \ldots \ldots \ldots 330$

6.8. L'espace des fonctions à déformations mesures........... 341



6.10 . Fonctions de mesure .......................... 350

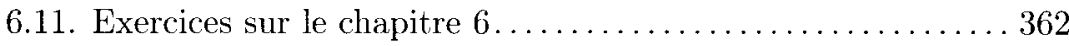

7. Sur l'inégalité de Korn dans $L^{p} \ldots \ldots \ldots \ldots \ldots \ldots \ldots \ldots \ldots \ldots \ldots \ldots \ldots$

7.1. Harmonicité. Moyennes. Fonction maximale de Hardy . . . . . 374

7.2. Transformation de Hilbert dans $\mathbb{R} \ldots \ldots \ldots \ldots \ldots \ldots \ldots . \ldots . \ldots . \ldots 38$

7.3. Les opérateurs de Riesz dans $\mathbb{R}^{N} \ldots \ldots \ldots \ldots \ldots \ldots \ldots \ldots \ldots 401$

7.4. Inégalité de Korn dans $W^{1, p}(\Omega), \Omega$ étant borné......... 409

7.5. Exercices sur le chapitre $7 \ldots \ldots \ldots \ldots \ldots \ldots \ldots \ldots \ldots \ldots 420$ 
Appendice sur la régularité........................437

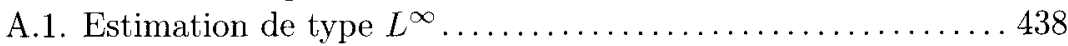

A.2. Estimations $W^{1, k}$ et $W^{1, \infty}$ dans le cas $p \geqslant 2 \ldots \ldots \ldots \ldots 443$

Bibliographie....................................457

Index $\operatorname{des}$ notations.................................

Index terminologique $\ldots \ldots \ldots \ldots \ldots \ldots \ldots \ldots \ldots \ldots \ldots \ldots \ldots$ 
